\title{
Circulatory Function Test with Electric Conductivity Method
}

\author{
Tatsuya Tomomatsu and \\ Kiichiro TANEMOTO
}

Kobe Medical College, Department of Internal Medicine, Division I.

Electric conductivity method is one of the indicator dilution methods which uses electrolyte solution as indicator. That is, blood flow and circulation time can be measured from the continuously recorded electric conductivity curve at the peripheral artery following certain amount of salt solution injection into the peripheral vein. Several basic problems of this method for its clinical use have already been investigated and reported in (Japan Journal of Resp. and Cir. 6: 613, 1958). In this report, following several applications of the method have been studied.

1. Measurement of cardiac output: Comparisons of the cardiac output measured with this method to that with Fick's principle, dye-dilution and radiocardiogram were made. The correlation coefficients were $0.920,0.965$ and 0.967, respectively. Moreover, using this method, simultaneous measurements of left and right cardiac output were followed with experimental adrenaline pulmonary edema in dog. It was shown that when pulmonary edema had developed, right cardiac output exceeded left one.

2. Determination of circulation time: From the analysis of electric conductivity curve, circulation time from the site of injection to the electrodes was determined. Particularly, accurrate pulmonary circulation time was obtained with the measurement of circulation time from the pulmonary artery to the left atrium and pulmonary blood volume was, also, calculated by multiplication of one second cardiac output to this time.

3. Measurement of venous return and its regional distribution: By application of this method on venous system with tributaries, venous return from various regions could be measured as already reported (Jap. Cir. J. 24: $1145,1297,1315,1960)$.

It has been emphasized that according to this method, venous return from various regions could be simultaneously measured in one case.

4. Measurement of ventricular residual volume: According to Bing's principle, it is possible to calculate ventricular residual volume by this method. In normal subjects and patients with cardiac or pulmonary disease right ventricular residual volume was measured. Furthermore, in dog induced adrenaline pulmonary edema simultaneous serial measurements of right and 
left ventricular residual volume were made. It had been demonstrated that both ventricular residual volume, particularly, left ventricular one, apparently increased about 4 or 5 times in fatal case.

5. Measurement of coronary blood flow: The early recirculated indicator dilution curve, which presumably was due to coronary blood return, could be recorded at either the pulmonary artery or right ventricle after injection of an indicator into the left ventricle. By this curve, coronary blood flow and coronary circulation time could be measured with dog and patient.

6. Diagnosis of congenital heart disease: It is possible to detect the existence and direction of shunt in congenital heart disease by the shape of dilution curve. At this time, in order to verify the diagnosis in doubtful case, serial dilution curves were recorded following Regitine $10 \mathrm{mg}$. intravenous injection or during Valsalva's maneuver.

7. Measurement of valvular regurgitant flow: In dog with experimentally produced mitral insufficiency, regurgitant flow could be measured by recording the curve at the left atrium after indicator injection into left ventricle. Possibility of the qualitative and quantitative determination of regurgitant flow had been discussed.

8. Determination of serum total base: With measurement of serum electric conductivity by Sunderman's method, rough estimation of serum total base could be made.

In conclusion, from these results, it was emphasized that this method is one of the most available means in cardiocirculatory function tests offering better evaluation. 